\title{
Trends in Anti-epileptic Drug Development
}

\author{
LENNART GRAM
}

Dianalund Epilepsy Hospital, Denmark

\section{Summary}

Several avenues are being explored in the development of new anti-epileptic drugs (AEDs).

For a number of years efforts have been directed towards compounds which may augment neuronal inhibition, and these efforts have resulted in the development of several valuable drugs. More recently, increased attention has been focused on the role which excitatory transmitters may play in epileptogenesis, and various substances which decrease excitation are currently being investigated at the preclinical level. Since considerable potential still resides in several of the drugs already on the market, resources have been spent on trying to modify/improve the chemical formula of some of these substances, and a number of new drugs has emerged as a result of this approach.

The major accomplishments reviewed here in the development of new anti-epileptic drugs suggest that even more successful advances may be achieved in the near future.

\section{Introduction}

During the last few decades major advances have been achieved in antiepileptic treatment. Several factors have contributed to this development (Table 1).

TABLE 1. Factors improving the treatment of epilepsy

1. Therapeutic drug monitoring

2. New treatment strategies (monotherapy)

3. Improved classification of seizure types/epilepsies

4. Introduction of new drugs

The monitoring of serum concentrations of anti-epileptic drugs, which was introduced 30 years ago (Buchthal et al., 1960), has improved our understanding of the importance of pharmacokinetics (Hvidberg and Dam, 1976). Monitoring of serum concentrations has made it possible to use the individual drug much more rationally than before and to test each drug to its full 
potential. Furthermore, it has also led to the idea of treating only with a single drug whenever possible, so-called monotherapy (Reynolds et al., 1976). This treatment strategy represents a significant departure from former therapeutic principles and has made for an improvement in seizure control, as well as a dramatic reduction in toxicity for many patients (Shorvon and Reynolds, 1979).

Classification of seizure types and of the epilepsies in general (Commission on Classification and Terminology, 1981; 1989) has also made great strides forward, thereby not only allowing epileptologists to express themselves in a universal language, but also enabling treatment results to be improved. Juvenile myoclonic epilepsy is a good example. The most disturbing seizure type in this syndrome is the convulsion. Today we know that, even though this seizure type normally responds quite well to treatment with carbamazepine or phenytoin, these drugs are of very limited value when convulsions occur in the context of this syndrome. In such cases, the patient should be treated with valproate, which is the drug of choice (Delgado-Escueta and Enrile-Bacsal, 1984).

Another important implication of the improved classification system is an increased understanding of how long anti-epileptic treatment should be continued. Again, juvenile myoclonic epilepsy serves as a good example: today we know that, contrary to almostall other types of epilepsy, this syndrome shows a very high recurrence rate when treatment is discontinued; for this reason, even when seizure control has been satisfactory for an extended period, treatment should not be discontinued in juvenile myoclonic epilepsy (Janz, 1985).

Some new drugs have come on to the market. Unfortunately, the ideal drug (Table 2) has yet to appear. Consequently, all the drugs available today represent compromises with regard to one or more of the points presented in the table. The key problem is twofold: on the one hand, we are still unable to control seizures in all patients; on the other, many patients who obtain complete or almost complete seizure control do so only at the expense of disturbing side-effects. The renewed interest in surgical treatment of epilepsy is a good therapeutic option for a minority of patients with drug-resistant epilepsy (Dam et al., 1988). However, there is clearly a need for developing new, more effective, and hopefully less toxic anti-epileptic drugs. Some of the avenues currently being explored will be briefly reviewed.

TABLE 2. The ideal anti-epileptic drug

1. Elicits complete seizure control in all patients

2. Is effective against all seizure types

3. Does not produce side-effects

4. Is easy to monitor (allowing infrequent visits by the patient)

5. Can be administered once or twice daily

6. Is inexpensive 


\section{GABAergic substances}

Gamma-aminobutyric acid (GABA), being the major inhibitory transmitter in the CNS, has attracted much interest over the years. It is evident that augmentation of suboptimal inhibition would be a possibility in the treatment of epileptic seizures (Meldrum, 1975).

A number of possibilities exists for manipulating the GABA system (Table 3). Today no drugs are available which increase the synthesis or the release of GABA. Until now the development of GABA agonists or prodrugs have not proved successful. Two compounds, THIP and progabide, have demonstrated either negative or only marginal benefits in the treatment of epilepsy (Petersen et al., 1963; Dam et al., 1983). GABA itself is unable to cross the blood-brain barrier, because it is not lipophilic. A single preliminary clinical report on treatment with liposome-entrapped GABA has appeared, reporting seizure reduction in epileptic patients (Loeb et al., 1987). Whether this is a treatment principle which should be further explored is at the moment uncertain.

TABLE 3. Theoretical possibilities for augmenting GABAergic inhibition

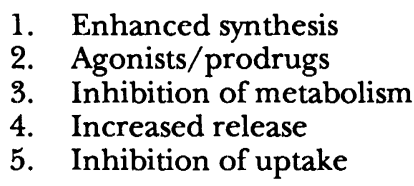

The most encouraging results have been obtained when trying to inhibit the metabolism of GABA. The major enzyme involved in the degradation of GABA is GABA-transaminase. Today, two drugs are available which influence this enzyme, valproate and vigabatrin. Due to the multiplicity of actions of valproate in the CNS, it is uncertain to what extent the influence on GABAtransaminase is responsible for the clinical effect of this drug (Gram, 1988). However, with regard to vigabatrin, which is very specific in its action, there seems to be little doubt that the mechanism responsible for the anti-epileptic effect of this compound is the irreversible inhibition of GABA-transaminase (Gram et al., 1989).

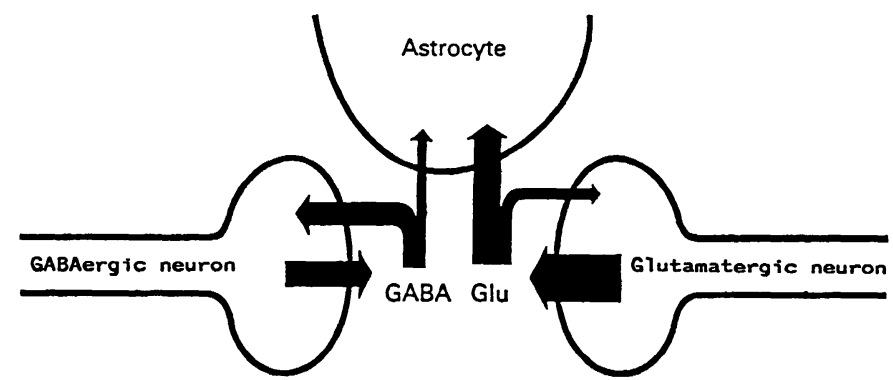

FIG. 1. Schematic diagram showing distribution of GABA and glumate between neuron and astrocyte (glial cell). 
Another possibility for influencing the GABA system would be to interfere with the uptake of GABA, which is taken up in both the neuron and in the glial cell (Fig. 1). The width of the arrows in the figure illustrates roughly the distribution between the amount of GABA taken up by the two cell types. Today we know that GABA which enters the neurons is re-used (Gram et al, 1988). However, GABA which enters the glia (astrocyte) is immediately degraded and lost for transmitter purposes. Consequently, it seems logical to try to develop glia-selective uptake inhibitors. This has already been done in the laboratory (Krogsgaard-Larsen et al.,1987), but the substances so far synthesized have two shortcomings: they do not cross the blood-brain barrier; and they exhibit toxicity problems in the animal species in which they have been tested. On the other hand, they demonstrate anticonvulsant properties. However, this has been found to be the case even for some non-selective GABA-uptake inhibitors, one of which is close to clinical testing (Novo Nordisk/Abbott, personal communication).

\section{Glutamatergic substances}

An increasing awareness exists today that only some forms of epilepsy are due to disturbances in the function of the GABA system. This inhibitory transmitter substance cannot therefore be regarded as the key to understanding epileptogenesis as such. Consequently, increased attention has been focused on the role which excitatory transmitters may play in the development of epilepsy. To reduce excitation seems as rational an approach as the concept of augmenting inhibition (Meldrum, 1984). It might perhaps even have additional unknown advantages. Several well established anti-epileptic drugs are known to influence excitatory systems (Schechter, 1978; Leach et al., 1986), the most important transmitter being glutamate.

It follows that there would be a number of different ways to influence excitatory systems (Table 4). So far, only some of these possibilities have been investigated.

TABLE 4. Theoretical possibilities for decreasing glutamatergic excitation

1. Decreased synthesis

2. Antagonists

3. Decreased release

4. Increased uptake

Apparently glutamate has three different receptors, named according to their preferred ligands, quisqualate, N-methyl-D-aspartate (NMDA), and kainate. With regard to epilepsy, it is the NMDA receptor which is of interest (Meldrum, 1984). A number of antagonists for this receptor has shown pronounced anticonvulsant properties. One substance, MK-801, has reached the clinical trial stage (Troupin et al., 1986). However, this is an area where intense pre-clinical research activity is taking place in a number of pharmaceutical companies.

Lamotrigine is a drug which is currently undergoing Phase III clinical trials. 
Controlled trials suggest an effect in therapy-resistant epilepsy (Jawad et al., 1989 ). Originally this compound was synthesized with the aim of producing new folate antagonists, but, although lamotrigine has a weak antifolate effect, subsequent studies seem to indicate that it decreases the release of glutamate (Leach et al, 1986). While it is not possible at the moment to conclude with absolute certainty that this is the mechanism responsible for its anti-epileptic effect, it is an interesting observation.

In contrast to GABA, only small amounts of glutamate are taken up in the neurons (Fig. 1). The vast majority enters the glial cells, resulting in degradation. Consequently, in developing new anti-epileptic compounds, substances which augment glutamate uptake should, at least from a theoretical point of view, be of value.

\section{Structural modification of existing drugs}

A completely different avenue for developing new anti-epileptic drugs would be to synthesize structural analogues of existing drugs. Since some very valuable drugs have already been in use for many years, the idea of trying to manipulate the chemical formula of these drugs has much to recommend it, the aim being to develop substances which represent an improvement over the parent compounds. This approach has been tried for a number of antiepileptic drugs, of which only three will be mentioned.

Phenobarbitone is a very effective anti-epileptic drug. However, the paramount problem is toxicity in the form of pronounced sedation. Eterobarb is a methyl derivative of phenobarbitone, which was synthesized several years ago. According to existing open and controlled studies, eterobarb seems to be more effective and to produce less sedation than phenobarbitone (Gallagher et al., 1975; Mattson et al., 1976) - this despite the fact that eterobarb is metabolised, partly via another metabolite, to phenobarbitone. With regard to the claimed superior clinical effect, what makes the interpretation of these studies difficult is the fact that treatment with eterobarb produces higher levels of phenobarbitone than does treatment with the parent substance itself. This could explain the observed difference in clinical effect.

Diazepam, nitrazepam, and clonazepam are recognized anticonvulsant benzodiazepines. However, they are all fraught in differing degrees with the problem of tolerance development. These substances are known as 1.4 benzodiazepines, indicating the positions of the carbon atoms in the heterocyclic ring. Manipulating the position of the carbon atoms has provided us with a 1.5 benzodiazepine, clobazam. Although this compound is well established as an anti-epileptic drug and is clearly less toxic than clonazepam, the problem of tolerance development is unfortunately very common with clobazam, limiting the value of the drug (Allen et al., 1985).

Oxcarbazepine represents one of the very successful manipulations of chemical formula. The compound is a keto-derivative of carbamazepine and has been subjected to extensive clinical testing, including controlled comparative evaluation versus carbamazepine (Dam et al., 1989; Reinikainen et al., 1987). The two drugs were shown to be comparable to carbamazepine with regard to clinical effect. However, with regard to toxicity, there was a significant 
difference in favour of oxcarbazepine, which may possibly be ascribed to differences in the metabolism of the two drugs. The degradation of oxcarbazepine does not involve the formation of an epoxide metabolite, and current evidence suggests that at least some of the toxicity of carbamazepine is caused by formation of the 10,11-epoxide. A further advantage of oxcarbazepine is that it seems to produce less enzyme induction than carbamazepine (Brodie et al., 1989).

Another possibility for detecting new anti-epileptic compounds is to screen newly synthesized substances for anticonvulsant properties, as is practised in the drug industry. However, there has been a declining interest in producing new anti-epileptic drugs. For this reason, the National Institutes of Health in the 1970s established the Antiepileptic Drug Development Program (Porter et al., 1984) to stimulate and combine efforts both from industry and from academic research. The result has been a number of new substances, which at the moment are approaching or undergoing clinical trials.

The major accomplishments reviewed here with regard to the development of new anti-epileptic drugs form the basis of our hope that even more successful advances may be achieved in the near future.

\section{References}

Allen, J.W., Oxley, J. and Trimble, M. (1985). Development of tolerance to the anticonvulsant effect of clobazam. Journal of Neurology and Neurosurgical Psychiatry, 48, 284-285.

Brodie, M.J., Larkin, J.G. and McKee, P.J. (1989). Is oxcarbazepine an enzyme inducer in man? 18th Epilepsy International Congress, New Delhi, 1989 (Abstract No. 107).

Buchthal, F., Svensmark, O. and Schiller, P.J. (1960). Clinical and electroencephalographic correlation with serum levels of diphenylhydantoin. Archives of Neurology, 2, 624-630.

Commission on Cilassification and Terminology of the International League Against Epilepsy (1981). Proposal for revised clinical and electroencephalographic classification of epileptic seizures. Epilepsia, 22, 489-501.

Commission on Classification and Terminology of the International League Against Epilepsy (1989). Proposal for revised classification of epilepsies and epileptic syndromes. Epilepsia, 30, 389-399.

Dam, M., Ekberg, R., Loynig, Y., Waltimo, O. and Jakobsen, K. (1989). A double-blind study comparing oxcarbazepine and carbamazepine in patients with newly diagnosed previously untreated epilepsy. Epilepsy Research, 3, 70-76.

Dam, M., Gram, L., Philbert, A., Hansen, B.S. and Lyon, B.B. (1983). Progabide: A controlled trial in partial epilepsy. Epilepsia, 24, 127-134.

Dam, M., Gram, L. and Schmidt, D. (1988). Surgical treatment of epilepsy. Acta Neurologica Scandinavica, 78, Suppl. 117.

Delgado-Escueta, A.V. and Enrile-Bacsal, F. (1984). Juvenile myoclonic epilepsy of Janz. Neurology, 34, 285-294.

Gallagher, B.B., Baumel, I.P., Woodbury, S.G. and Dimicco, J.A. (1975). Clinical evaluation of eterobarb, a new anticonvulsant drug. Neurology, 25, 399-404.

Gram, L. (1988). Experimental studies and controlled clinical testing of valproate and vigabatrin. Acta Neurologica Scandinavica, 78, 241-270.

Gram, L., Larsson, O.M., Johnsen, A. and Schousboe, A. (1988). Effect of valproate, vigabatrin and aminooxyacetic acid on release of endogenous and exogenous GABA from cultured neurons. Epilepsy Research, 2, 87-95.

Gram, L., Larsson, O.M., Johnsen, A. and Schousboe, A. (1989). Experimental studies on the influence of vigabatrin on the GABA system. British Journal of Clinical Pharmacology, 27, Suppl. 1, 13S-19S. 
Hvidberg, E.F. and Dam, M. (1976). Clinical pharmacokinetics of anticonvulsants. Clinical Pharmacokinetics 1, 161-168

Janz, D. (1985). Epilepsy with impulsive petit mal (juvenile myoclonic epilepsy. Acta Neurologica Scandinavica, 72, 449-459.

Jawad, S., Richens, A., Goodwin, G. and Yuen, W.C. (1989). Controlled trial of lamotrigine (Lamictal) for refractory seizures. Epilepsia, 30, 356-363.

Krogsgaard-Larsen, P., Falch, E., Larsson, O.M. and Schousboe, A. (1987). GABA uptake inhibitors: Relevance to antiepileptic drug research. Epilepsy Research, 1, 77-93.

Leach, M.J., Harden, C.M. and Miller, A.A. (1986). Pharmacological studies on lamotrigine, a novel potential antiepileptic drug. II. Neurochemical studies on the mechanism of action. Epilepsia, 27, 490-497.

Loeb, C., Benassi, E., Bo, G.P., Cocito, L., Maffini, M. and Scotto, P. (1987). Preliminary evaluation of the effect of GABA and phosphatidylserine in epileptic patients. Epilepsy Research, 3, 209-212.

Mattson, R.H., Williamson, P.D. and Hanah, E. (1976). Eterobarb therapy in epilepsy. Neurology, 26, 1014-1017.

Meldrum, B.S. (1975). Epilepsy and gamma-aminobutyric acid-mediated inhibition. International Review of Neurobiology, 17, 1-36.

Meldrum, B.S. (1984). Amino acid neurotransmitters and new approaches to anticonvulsant drug action. Epilepsia, 25, Suppl. 2, S140-S149.

Petersen, H.R., Jensen, I. and Dam, M. (1983). THIP, a single-blind controlled trial in patients with epilepsy. Acta Neurologica Scandinavica, 67, 114-117.

Porter, R.J., Cereghino,J.J., Gladding, G.D., Hessie, B.J., Kupferberg, H.J., Scoville, B. and White, B.G. (1984). Antiepileptic drug development program. Cleveland Clinic Quarterly, 51, 293-305.

Reinikainen, K.J., Keraanen, T., Halonen, T., Koumlainen, H. and Riekkinen, P.J. (1987). Comparison of oxcarbazepine and carbamazepine: A double-blind study. Epilepsy Research, 1, 284-289.

Reynolds, E.H., Chadwick, D. and Galbraith, A.W. (1976). One drug 'phenytoin' in the treatment of epilepsy. Lancet, i, 923-926.

Schechter, P.J., Tranier, Y. and Grove, J. (1978). Effect of $n$-dipropylacetate on amino acid concentrations in mouse brain: Correlation with anticonvulsant activity. Journal of Neurochemistry, 31, 1325-1327.

Shorvon, S.D. and Reynolds, E.H. (1979). Reduction in polypharmacy for epilepsy. British Medical Journal, 2, 1023-1025.

Troupin, A.S., Mendins, J.R., Cheng, F. and Risinger, M.W. (1986). MK-801. In "New Anticonvulsant Drugs." (Eds B.S. Meldrum and R.J. Porter), pp. 191-201.John Libbey, London.

Watkins, J.C. and Evans, R.H. (1981). Excitatory amino acid transmitters. Annual Review of Pharmacology and Toxicology, 21, 165-204. 


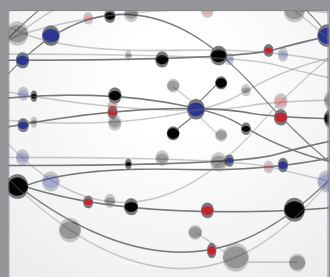

The Scientific World Journal
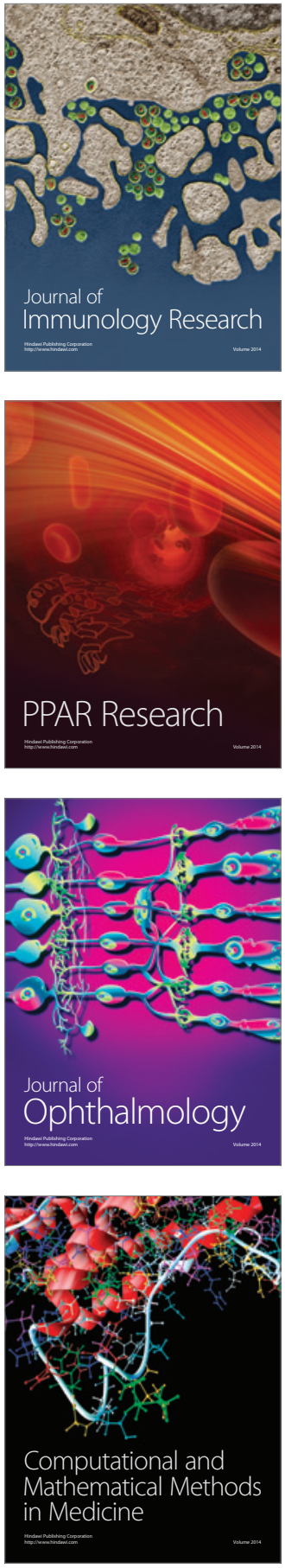

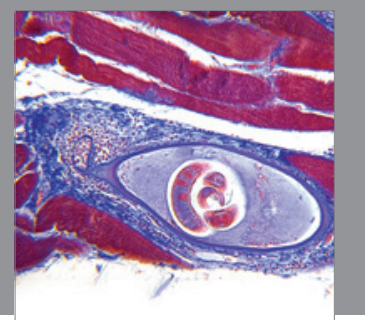

Gastroenterology

Research and Practice
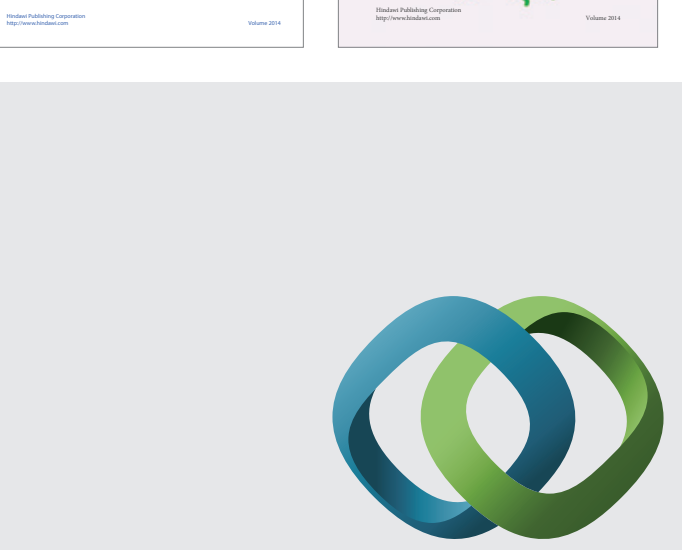

\section{Hindawi}

Submit your manuscripts at

http://www.hindawi.com
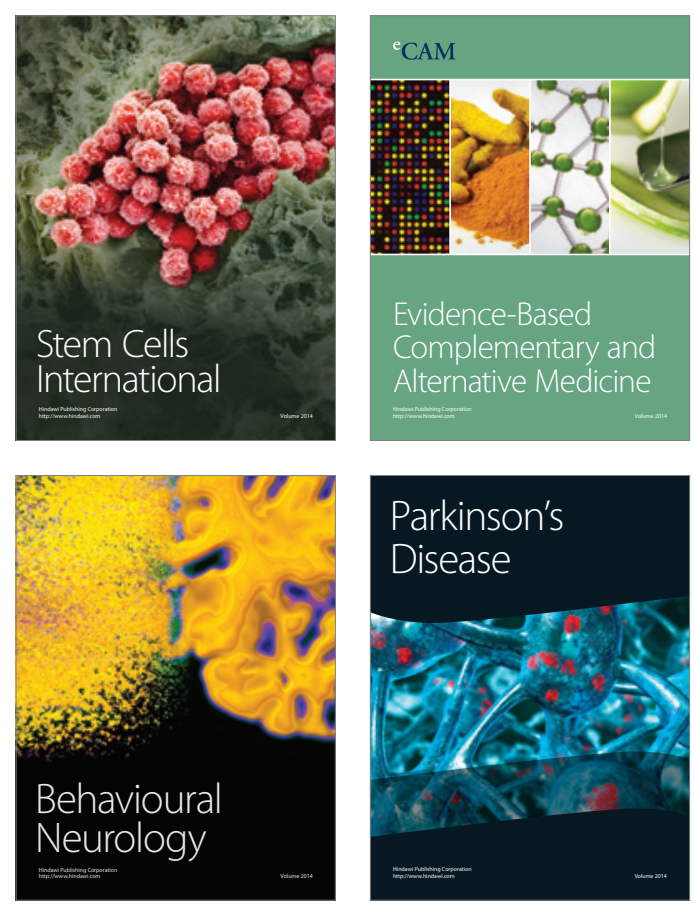

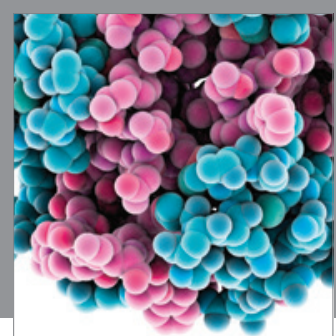

Journal of
Diabetes Research

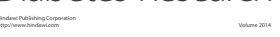

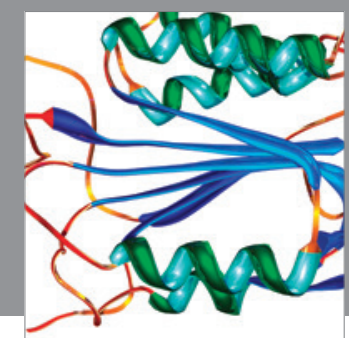

Disease Markers
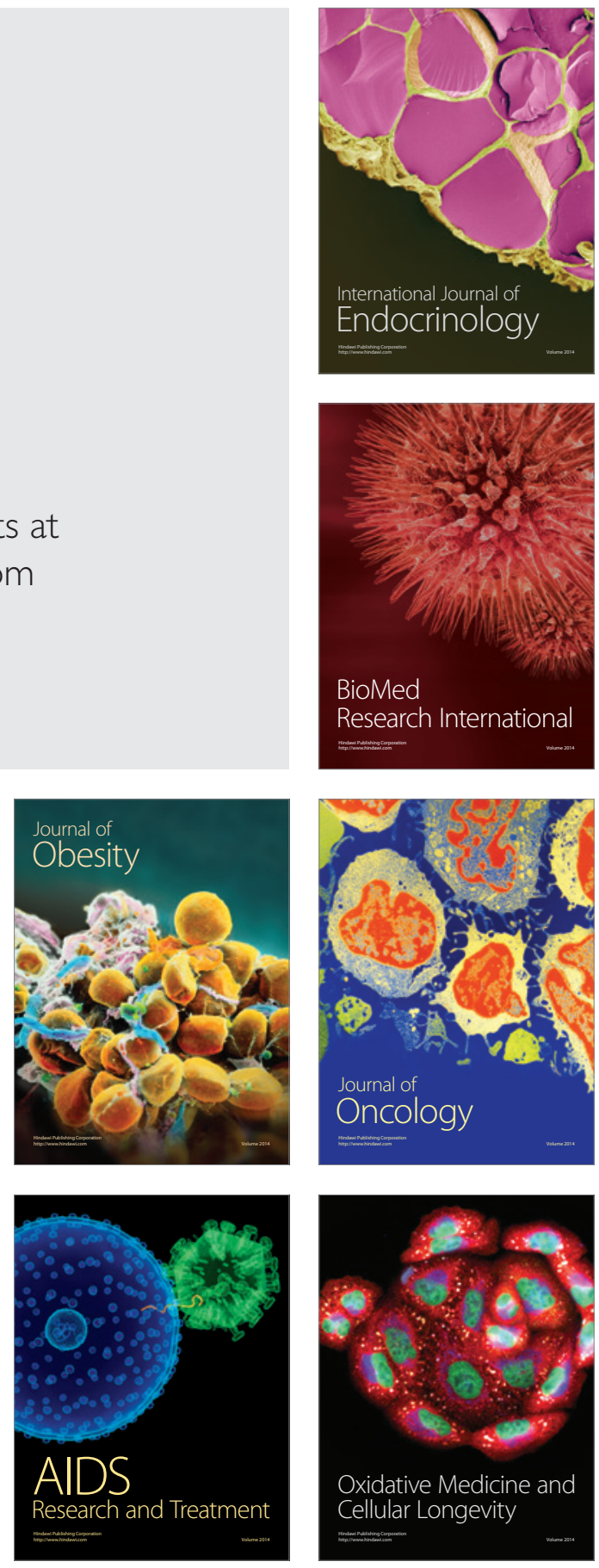